\title{
Neonatal Follow Up Study: A Retrospective Chart Review of Developmental Outcomes
}

Heather Wolfe, $\mathrm{MD}^{1}$, Denise Gilham, NP², Susan Stace, RN, CPN ${ }^{3}$, Uchechukwu Emili, BS ${ }^{4}$

${ }^{1}$ Lutheran Hospital, ${ }^{2}$ Indiana University School of Medicine

Background and Hypothesis: According to the CDC, Preterm birth is defined as a baby born before 37 weeks of gestation. ${ }^{1}$ In 2016 preterm birth affected 1 in every 10 infant born in the United States. ${ }^{1}$ Preterm birth has been known to cause many birth defects along with developmental delays and has attributed to $17 \%$ of all infant death. Neonatal Follow Up clinics or programs have been instrumental in helping infants catch up developmentally. ${ }^{3} \mathrm{We}$ believe that with the NFC, we will be able to develop a standard for preterm babies and the normalization of their development.

Experimental Design or Project Methods: A retrospective chart review was conducted to determine the developmental outcome of infants that were born prior to 37 weeks' gestation. Infants were divided into extreme, very, and moderate preterm cohorts. Test of Infant Motor Performance (TIMP), Ages and Stages Questionnaire (ASQ), Bayley Scales of Infant Development (BSID) and Modified Checklist for Autism in Toddlers (M-CHAT) scores were used to determine a standard for when preterm babies in each cohort normalize developmentally.

Results: Analysis of the data showed that the developmental turning point for each cohort varies. Within the extreme preterm cohort, one can see that there was no clear turning point for development. For the very preterm group, by TIMP 2, one could see a turning point and a decrease in delays. Lastly, moderately preterm cohort, the number of delays was seen tot decrease from the administration of TIMP 2 and continue in this trend.

Conclusion and Potential Impact: Through the administration of these developmental tests, physicians are able to track the development of preterm babies. We concluded that with the proper follow up, one can see an improvement in development as early as the administration of TIMP 2.

1) Preterm Birth https://www.cdc.gov/reproductivehealth/MaternallnfantHealth/PretermBirth.htm

2) Suave, R., \& Lee, S. K. (2006). Neonatal follow-up programs and follow-up studies: Historical and current perspectives. Paediatrics and Child Health. http://doi.org/10.1093/pch/11.5.267

3) Voller, S. M. B. (2018). Follow-Up Care for High-Risk Preterm Infants. Pediatric Annals, 47(4).

http://doi.org/10.3928/19382359-20180325-03 